\title{
ОТНОШЕНИЕ К ИЗМЕНЕНИЯМ И ТОЛЕРАНТНОСТЬ К НЕОПРЕДЕЛЕННОСТИ КАК ПРЕДИКТОРЫ АДАПТИВНОСТИ И АДАПТАЦИОННОЙ ГОТОВНОСТИ
}

\author{
Раиль М. Шамионов \\ Саратовский национальный исследовательский государственный уни- \\ верситет имени Н. Г. Чернышевского, г. Саратов, Российская Федерация \\ E-mail: shamionov@info.sgu.ru
}

Публикация подготовлена в рамках поддержанного РГНФ научного проекта № 15-06-10624

Введение. Автором доказывается актуальность изучения детерминант социально-психологической адаптивности и адаптационной готовности и их взаимосвязи. Новизна исследования заключается в получении новых данных о предикторах и уровне предикции адаптивности и адаптационной готовности личности, структурной модели с установлением путей для готовности к изменениям, адаптации, отношения к неопределенной ситуации и адаптивности личности.

Методы. В разделе описываются использованные методики: оценки характеристик толерантности к неопределенности Д. МакЛейна в адаптации Е. Н. Осина, методика социально-психологической адаптивности К. Роджерса - Р. Даймонда в адаптации А. К. Осницкого, а также разработанные оригинальные шкалы для оченки адаптационной готовности и готовности к изменениям. Представлены данные о выборке: в исследовании приняли участие 75 человек обоих полов (мужчин $38 \%$, средний возраст 26,8 лет).

Результаты исследования и их обсуждение. Проведена классификация исходного множества параметров на группы, и установлены соотношения между ними. Установлено, что готовность к изменениям, адаптационная готовность, толерантность к неопределенности и адаптивность образуют единый симптомокомплекс, характеризующийся сложными межфункциональными взаимосвязями. На основании структурного моделирования выделены пути для готовности к изменениям, адаптации, отношения к неопределенной ситуации и адаптивности личности.

В заключение делается вывод о том, что готовность к изменениям играет опосредующую роль во взаимосвязях адаптационной готовности и отношения к неопределенности; адаптивность, адаптационная 
готовность и отношение к неопределенности образуют кольцевую взаимосвязь. Сочиально-демографические характеристики образуют комплекс предикторов социально-психологической адаптивности, но не связаны с адаптационной готовностью. Отношение к неопределенности снижает социально-демографическую детерминацию и играет значительную роль в формировании адаптивности личности.

Ключевые слова: адаптационная готовность, адаптивность, личность, отношение, толерантность к неопределенности, готовность к изменениям, детерминанты, кластерный анализ, структура, структурное моделирование

\section{Основные положения:}

> на основе кластерного анализа выявлена более весомая значимость адаптационной готовности и толерантности к неопределенности для сочиально-психологической адаптации личности;

в результате регрессионного анализа установлено, что отношение к неопределенности играет значительную роль в формировании адаптивности личности;

> в результате структурного моделирования показаны направления связей между адаптивностью, адаптационной готовностью и толерантностью к неопределенности: адаптивность оказывает существенное влияние на адаптационную готовность прямо, а адаптационная готовность на нее - опосредованно, через отношение к неопределенной ситуации.

Для цитирования: Шамионов Р. М. Отношение к изменениям и толерантность к неопределенности как предикторы адаптивности и адаптационной готовности // Российский психологический журнал. - 2017. - Т. 14, № 2. - С. 90-104. 


\title{
ATTITUDE TO CHANGE AND TOLERANCE TO UNCERTAINTY AS PREDICTORS OF ADAPTABILITY AND ADAPTIVE READINESS
}

\author{
Rail M. Shamionov \\ Saratov State University, Saratov, Russian Federation \\ E-mail: shamionov@info.sgu.ru

\section{Acknowledgments} \\ Supported by the Russian Humanitarian Science Foundation project \\ no. $15-06-10624$
}

Introduction. Studying the determinants of socio-psychological adaptability and adaptive readiness, as well as their relationship, is an important issue of recent research. This study presents new data on predictors and the level of prediction of the person's adaptability and adaptive readiness, the structural model with the ways for readiness to change, adaptation, the attitude to uncertain situations, and the person's adaptability.

Methods. The study used the following techniques: (a) the estimation of the characteristics of tolerance to uncertainty by D. McClane in E. N. Osin's modification; (b) the technique for diagnostics of socio-psychological adaptability by K. Rogers and R. Diamond in A. K. Osnitsky's modification; (c) the developed original scales for the assessment of adaptive readiness and readiness to change. The study involved 75 women and men (men - 38\%, mean age - 26.8 years).

Results and discussion. The study presented the classification of the original set of parameters into groups and associations between them. Adaptive readiness, readiness to change, tolerance to uncertainty, and adaptability form a united symptom complex with complex cross-functional interrelations. On the basis of structural modeling the study revealed the ways for readiness to change, adaptation, the attitude to uncertain situations, and the person's adaptability.

In conclusion: readiness to change mediates the relationship between adaptive readiness and the attitude to uncertainty. Adaptability, adaptive readiness and the attitude to uncertainty form a circular relationship. Socio-demographic characteristics form a complex of predictors of socio-psychological adaptability. They are not associated with adaptive readiness. The attitude to uncertainty reduces sociodemographic determination and plays a significant role in the formation of the person's adaptability. 
Keywords: adaptive readiness, adaptability, personality, attitude, attitude to uncertainty, readiness to change, determinants, cluster analysis, structure, structure modeling

\section{Highlights}

- The cluster analysis revealed the higher significance of adaptive readiness and tolerance to uncertainty for the person's socio-psychological adaptation.

- The regression analysis revealed that the attitude to uncertainty is important for the person's adaptability.

> The results of structural modeling showed the directions of relationship between adaptability, adaptive readiness, and tolerance to uncertainty. Adaptability has a significant direct influence on adaptive readiness, while adaptive readiness influences adaptability indirectly, through the attitude to uncertain situations.

For citation: Shamionov R. M. Attitude to change and tolerance to uncertainty as predictors of adaptability and adaptive readiness. Rossiiskii psikhologicheskii zhurnal - Russian Psychological Journal, 2017, V. 14, no. 2, pp. 90-104 (in Russian).

Original manuscript received 04.12.2016

\section{Введение}

Изучение детерминант социально-психологической адаптивности и адаптационной готовности и их взаимосвязи необходимо для разработки средств оперативной помощи человеку, находящемуся в ситуации приспособления, для построения системы социально-психологической поддержки личности в ситуации социальных или жизненных изменений, в которые она включается, и сквозь которые преломляются ее стабильные и динамические характеристики.

Необходимо особо подчеркнуть, что адаптационная готовность личности (в отличие от адаптивности) является эффектом ее социализации. Поэтому она более чувствительна к внешним условиям и может быть сформирована через систему социально-психологической поддержки. Вместе с тем, формирование адаптационной готовности основано на собственном опыте адаптации точно так же, как и на тех внешних представлениях, ценностях и установках, которые складываются стихийно. Известный исследователь в области адаптации личности А. А. Налчаджян отмечает, что адаптивные комплексы (механизмов), актуализируясь в сходных ситуациях и функционируя, «закрепляются в структуре личности, становятся подструктурами ее характера» [1, с. 27]. Иначе говоря, эти механизмы адаптации, закрепляясь, создают определенный «запас уверенности» в возможности адаптации 
к различным ситуациям, и тем самым - готовность к адаптации. Его действие демонстрирует исследование С. Т. Джанерьян и И. Н. Астафьевой, в котором показано, что отдельные характеристики социальной фрустрации необязательно связаны с социально-психологической адаптивностью [2]. Это свидетельствует в пользу того, что неудовлетворенность личности какой-либо стороной бытия еще не выводит ее на дезадаптацию. Очевидно, адаптационная готовность, будучи доадаптивной формой саморегуляции, «гасит» некоторые проявления фрустрации.

Адаптационная готовность определена нами как состояние индивида, сопровождающееся психическим напряжением, позволяющим сохранить настрой личности на приспособление к значимой с точки зрения реализации потребностей ситуации, и включающее представление о желаемом (успешном) его исходе, а также допустимых средствах и действиях (на приспособление) [3].

Исследования детерминант адаптивности и адаптационной готовности сосредоточены вокруг ряда областей - личностных свойств и, прежде всего, субъектных свойств и социального интеллекта [4], готовности к риску [5], системных компонентов саморегуляции личности [6], ценностно-смысловых образований [7], Я-концепции и социокультурного пространства [8], эмоционального выгорания [9], оценки сложности ситуации и представления об эмоциональной саморегуляции [10], влияния социальной поддержки и удовлетворенности потребностей на внутригрупповую идентичность и адаптацию при смене групп членства [11], и др.

Кроме того, исследователи находятся в непрерывном поиске по вопросу о соотношении адаптационной готовности и адаптации как процесса и адаптированности как его результата $[12,13]$. Отметим, что до сих пор эти изыскания не привели к однозначному результату. Особый вопрос также возникает относительно влияния (взаимовлияния) этих переменных. Поэтому анализ различных психологических и социально-психологических характеристик личности как факторов адаптационной готовности вынуждает обращаться одновременно и к адаптивности как характеристике, отражающей внутреннее равновесие личности, субъективную адаптированность, а не установку (пусть и интегральную) на адаптацию, которая в ряде случаев может и «не сработать», наткнувшись на ряд барьеров или фрустраторов. Кроме того, было бы небезынтересно установить не просто корреляционную связь, что выявлялось неоднократно в исследованиях, но направленность этой связи, ее специфику.

Актуализация адаптационной готовности связана с изменением ситуации, с контролем стабильности в ней. Поэтому важнейшим обстоятельством в ее реализации является готовность к изменениям и толерантность 
к неопределенности $[14,15,16]$. Предсказуемые изменения ситуации и неопределенность в разной степени способны нарушить установившийся баланс, динамическое равновесие в системе «личность - среда». Поэтому возникает вопрос о специфике взаимосвязей между характеристиками адаптационного блока и отношения к изменению ситуации.

Цель данного исследования - провести анализ готовности к изменениям и толерантности к неопределенности как предикторов адаптивности и адаптационной готовности и установить направленности связей между изучаемыми характеристиками.

\section{Методы}

Характеристика выборки: в исследовании приняли участие 75 человек обоих полов (38\% мужчин) - M = 26,8; SD = 7,7, в том числе $48 \%$ семейных, $52 \%$ со средним профессиональным образованием, большинство (48,3\%) имеет средний уровень дохода по региону, 84\% христиан, 64\% горожан.

Для оценки адаптационной готовности и готовности к изменениям нами были разработаны шкалы. Из 14 шкал, первоначально выделенных, после их анализа и оценки экспертами, отбраковки для предъявления испытуемым осталось 10 шкал, каждая из которых предполагает оценку от 1 до 5 баллов по мере оцениваемой субъективной выраженности. Оценка надежности-согласованности проведена с помощью альфа Кронбаха. Для шкал адаптационной готовности при удалении пункта $a=0,78-0,82$. Анализ корреляций шкал адаптационной готовности и адаптивности составляет $r=0,562-0,622$, что относится к умеренно высокой корреляции с высокой значимостью $\mathrm{p}<0,001$. Факторный анализ, проведенный по всем шкалам, позволил выявить два отдельных фактора: по общей адаптационной готовности величина охватываемой дисперсии составляет 55,7\%; по готовности к изменениям - $28 \%$.

Для оценки характеристик толерантности к неопределенности использована методика Д. МакЛейна «Multiple Stimulus Types Ambiguity Tolerance Scale-І» в адаптации Е. Н. Осина [17]. Использованный вариант методики состоит из 18 вопросов и включает 5 шкал: отношение к новизне, отношение к сложным задачам, отношение к неопределенным ситуациям, предпочтение неопределенности, толерантность к неопределенности. Каждый пункт опросника оценивается от 1 до 7 баллов $(\alpha=0,79-0,89)$.

Для оценки параметров социально-психологической адаптивности использована методика К. Роджерса - Р. Даймонда в адаптации А. К. Осницкого [18]. Опросник включает 101 пункт, степень согласия с каждым оценивается испытуемым от 0 до 6 баллов. Опросник содержит 14 первичных шкал и 6 интегральных показателей: «Адаптация», «Принятие 
других», «Интернальность», «Самовосприятие», «Эмоциональная комфортность», «Стремление к доминированию» $(a=0,89-0,92)$.

Кроме основных показателей получены социально-демографические данные о выборке: пол, возраст, место проживания, этническая самоидентификация и религиозные предпочтения, уровень дохода и др., которые использованы также для включения в уравнение регрессии.

\section{Результаты исследования и их обсуждение}

С целью классификации и установления расстояния между переменными, отражающими адаптацию, адаптационную готовность, готовность к изменениям и толерантность к неопределенности, нами проведен иерархический кластерный анализ с использованием метода межгрупповых связей. Обратимся к результатам кластерного анализа. Как видно из представленной дендрограммы, все включенные в анализ переменные распределились на два основных кластера. Основные характеристики социально-психологической адаптации объединены в один кластер, а адаптационная готовность, готовность к изменениям и толерантность к неопределенности - в другой кластер.

Интересным представляется и то, что на низшем уровне иерархии образуются три кластера: первый состоит из адаптационной готовности, связанной с отношением к новизне и эскапизмом (уходом от проблем и принятием ответственности), и эта триада наиболее близка к готовности к изменениям; ближайший к нему второй кластер образуют отношение к сложным задачам, отношение к неопределенным ситуациям и толерантность к неопределенности, - наиболее близким к ним признаком является предпочтение неопределенности; наконец, третий кластер образуют адаптация, интернальность и принятие других с наиболее близким к ним признаком - самопринятием и эмоциональным комфортом. Из этих данных следует, что для проявлений адаптационной готовности, отношения к новизне и эскапизма наиболее важной выступает готовность к изменениям, для всего блока показателей толерантности к неопределенности - предпочтение неопределенности, а для социально-психологической адаптации, основанной на принятии других и ответственности - самопринятие и эмоциональная комфортность. Последнее, однако, находится под действием стремления к доминированию. Как видно из дендрограммы (рисунок 1), первые два описанных кластера выступают доминантными в отношении третьего, из чего следует более весомая значимость адаптационной готовности и толерантности к неопределенности для социально-психологической адаптации личности.

Обратимся к данным, отражающим детерминантный комплекс социальнопсихологической адаптивности и адаптационной готовности. 
Отн_к_новизне

Адаптац_готовность

Эскапизм

Готовность_к_изм

Отн_к_сложным_задачам

Отн_к_неопред_ситуациям Толерантность_к_неопредел

Предпочтение_неопредел

Адаптация_общая

Эмоц_комфортность

Интернальность

Принятие_других_общ

Самовосприятие

Стремление_к_доминированию

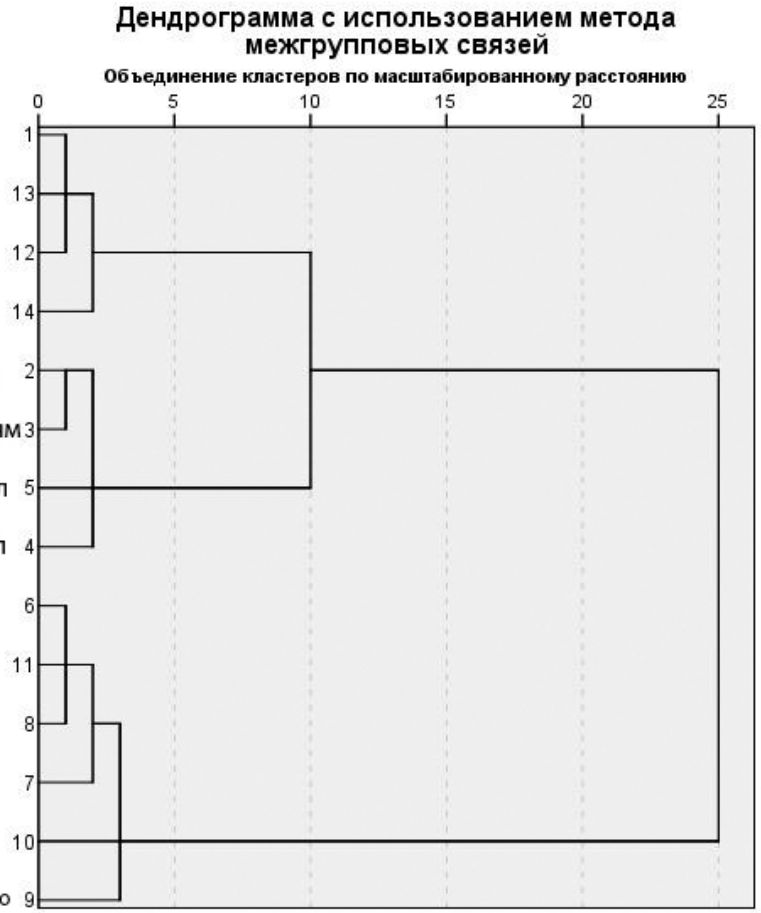

Рисунок 1. Дендрограмма по результатам кластерного анализа переменных по методу межгрупповых связей

Figure 1. Results of the cluster analysis of variables by the method of intergroup relationship (a dendrogram)

Регрессионный анализ с введением социально-демографических характеристик как предикторов адаптивности позволил установить весьма сильную зависимость ее вариаций. Причем доля дисперсии зависимой переменной (адаптивности) по каждому параметру примерно одинакова. В частности, предикторами являются доход $\left(\Delta R^{2}=0,08\right)$ [здесь и далее $\Delta \mathrm{R}^{2}$ - собственный вес показателя, его вклад в коэффициент множественной детерминации], возраст (отрицательно) $\left(\Delta \mathrm{R}^{2}=0,06\right)$, сила этнических чувств $\left(\Delta R^{2}=0,07\right)$, пол $\left(\Delta R^{2}=0,05\right)$, семейное положение $\left(\Delta R^{2}=0,07\right)$. В совокупности, эти показатели объясняют $32 \%\left(\Delta R^{2}=0,32 ; F=6,12, p<0,001\right)$ дисперсии адаптивности. Из этих данных следует, что социально-демографический портрет адаптивной личности включает относительно высокий 
доход, силу этнических чувств, наличие семьи. Мужчины характеризуются более высокой адаптивностью. Одним из весьма примечательных результатов здесь является место и роль силы этнических чувств. Введение в уравнение регрессии этой переменной позволило более дифференцированно рассмотреть социодемографические показатели как детерминанты адаптивности, поскольку без нее они зауживаются на двух параметрах, что позволяет предположить ее посредничество между социально-демографическими показателями и адаптивностью, а это, в свою очередь, свидетельствует о ее высокой значимости для всего процесса адаптации. Очевидно, сила этнических чувств говорит о достаточно явной идентификации, этнической определенности и (возможно) позитивности оценки своей этнической принадлежности, что и служит своеобразным плацдармом для согласования себя со своим социальным окружением.

Однако наиболее значимой детерминантой адаптивности выступает адаптационная готовность $\left(\Delta R^{2}=0,31\right)$ - комплекс установок в отношении своих возможностей и намерений в приспособлении к различным социальным ситуациям, которая при введении в регрессионное уравнение смещает социально-демографические показатели на второстепенную позицию. Аналогичный подход к регрессионному анализу с включением в качестве зависимой переменной адаптационной готовности не был успешным. Очевидно, адаптационная готовность является генетически вторичной по отношению к адаптивности. Данное предположение перекликается и с теоретическими взглядами М. В. Григорьевой, в соответствии с которыми адаптационная готовность личности рассматривается как новообразование в адаптационном процессе в системе «личность - среда» [12].

В отношении параметров толерантности к неопределенности имеются различия в весе регрессии. Так, $25 \%$ вариаций адаптационной готовности детерминированы показателем «отношение к неопределенным ситуациям» $\left(R^{2}=0,25 ; F=24,74, p<0,001\right)$. В то же время, лишь $14 \%$ вариаций готовности к изменениям детерминированы этой переменной $\left(\mathrm{R}^{2}=0,14\right.$; $\mathrm{F}=12,31, \mathrm{p}<0,001)$. Иначе говоря, отношение к неопределенным ситуациям служит влияющим фактором и адаптационной готовности и готовности к изменениям. Наконец, $21 \%$ вариаций адаптивности $\left(\mathrm{R}^{2}=0,21\right.$; $\mathrm{F}=19,21, \mathrm{p}<0,001)$ обусловлен отношением к неопределенности. Все эти данные свидетельствуют о весомой роли отношения к неопределенности в формировании готовности к изменениям, к адаптации и адаптивности. Очевидно, позитивное отношение к неопределенности, терпимость к ней, принятие ее, уверенность в том, что можно справиться с неопределенной ситуацией, - служат внутренним основанием для уверенности в возможности приспособления. Совершенно неслучайна в этой картине и связь между 
адаптивностью и готовностью к изменениям ( $r=0,227, p<0,01)$. Поскольку готовность к изменениям соотносится с готовностью к риску [19], данные результаты не кажутся случайными. Так, И. В. Арендачук показано, что лица, характеризующиеся высоким уровнем готовности к риску, демонстрируют и более высокий уровень социально-психологической адаптивности и других показателей адаптационного профиля личности [5].

Обратимся к результатам структурного моделирования (SEM). Как видно из таблицы 1, индекс пригодности свидетельствует о приемлемости данного варианта модели. Другие варианты взаимовлияния данных характеристик ухудшают свойства модели, что явилось основанием для их исключения.

Таблица 1.

\section{Данные о пригодности модели}

Table 1.

The suitability of the model

\begin{tabular}{|c|c|c|c|c|c|}
\hline$X^{2}$ & $\boldsymbol{d f}$ & $\boldsymbol{X}^{2 / \boldsymbol{d} \boldsymbol{f}}$ & $\boldsymbol{p}$ & $\boldsymbol{R M S E A}$ & CFI \\
\hline 0,149 & 1 & 0,15 & 0,699 & 0,000 & 0,99 \\
\hline
\end{tabular}

На рисунке 2 представлена диаграмма путей с соответствующими коэффициентами регрессии между различными характеристиками.

Как видно на рисунке 2, готовность к изменениям играет опосредующую роль во взаимосвязях адаптационной готовности и отношения к неопределенности. Между тем, адаптивность, адаптационная готовность и отношение к неопределенности образуют кольцевую взаимосвязь. При этом влияние адаптивности на адаптационную готовность более весомо. Из этих данных следует, что вопреки ранее сделанным предположениям о том, что адаптационная готовность оказывает влияние на процесс адаптации в большей степени, нежели адаптивность на адаптационную готовность [13], наличие хорошего опыта адаптации создает благоприятную картину для формирования адаптационной готовности, которая, в свою очередь обусловливает и готовность к изменениям, и отношение к неопределенной ситуации. Интересно и то, что готовность к изменениям не прямо оказывает влияние на адаптивность, но опосредованно, через отношение к неопределенности. Таким образом, адаптивность оказывает существенное влияние на адаптационную готовность прямо, а адаптационная готовность на нее - опосредованно, через отношение к неопределенной ситуации. 


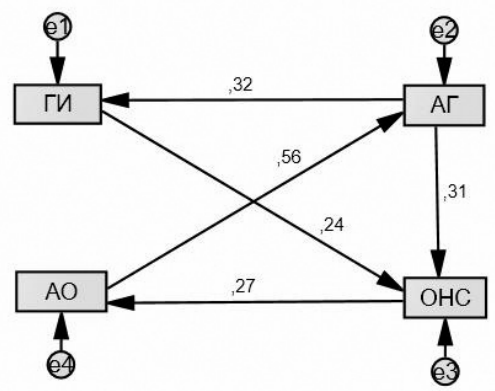

хи-квадрат=,149: $d f=1: p=, 699:$ RMSEA $=, 000$

Рисунок 2. Диаграмма путей для готовности к изменениям, адаптации, отношения к неопределенной ситуации и адаптивности личности (все стандартизированные оценки с уровнем значимости $\mathrm{p}<0,01$ )

Figure 2. Diagram of ways for readiness to change, adaptation, the attitude to uncertain situations, and the person's adaptability (correlation coefficients are significant at the 0.01 level)

Примечание. Приняты следующие сокращения: ГИ - готовность к изменениям, АГ - адаптационная готовность, АО - адаптивность общая, ОНС - отношение к неопределенной ситуации.

Note. The following abbreviations are used: $R C$ - readiness to change, $A R$ - adaptive readiness, GA - general adaptability, AUS - attitude to uncertain situations.

\section{Заключение}

Результаты проведенного эмпирического исследования позволяют утверждать, что готовность к изменениям, адаптационная готовность, толерантность к неопределенности и адаптивность образуют единый симптомокомплекс, характеризующийся сложными межфункциональными взаимосвязями. Исходя из результатов кластерного анализа, можно утверждать, что для проявлений адаптационной готовности, отношения к новизне и эскапизма наиболее важной выступает готовность к изменениям, для всего блока показателей толерантности к неопределенности - предпочтение неопределенности, 
а для социально-психологической адаптации, основанной на принятии других и ответственности, - самопринятие и эмоциональная комфортность.

Социально-демографические характеристики образуют комплекс предикторов социально-психологической адаптивности, но не связаны с адаптационной готовностью. Отношение к неопределенности снижает социально-демографическую детерминацию и играет значительную роль в формировании адаптивности личности. Наличие хорошего опыта адаптации создает благоприятную картину для формирования адаптационной готовности, которая, в свою очередь обусловливает и готовность к изменениям, и отношение к неопределенной ситуации.

\section{Литература}

1. Налчаджян А. А. Психологическая адаптация. - М. : Эксмо, 2010. - 268 с.

2. Джанерьян С. Т., Астафьева И. Н. Социальная фрустрированность выпускников школ (юношей и девушек) в связи с их индивидуально-психологическими особенностями // Актуальные проблемы гуманитарных и естественных наук. - 2015. - № 9-2. - С. 182-186.

3. Шамионов Р. М. Соотношение толерантности к неопределенности и адаптационной готовности личности к изменениям // Вестник Российского университета дружбы народов. Серия: Психология и педагогика. - 2016. - № 3. - С. 28-39.

4. Григорьева М. В. Роль социального интеллекта в процессе психологической адаптации старшеклассников // Теория и практика общественного развития. - 2015. - № 6. - С. 171-174.

5. Арендачук И. В. Личностный потенциал и социально-психологическая адаптированность как ресурс психологической готовности к риску // Известия Саратовского университета. Новая серия. Акмеология образования. Психология развития. - 2015. - Т. 4, № 3. - С. 219-226.

6. Бочарова Е. Е. Адаптационная готовность личности к ситуациям социальных изменений // Известия Саратовского университета. Новая серия. Акмеология образования. Психология развития. - 2015. - Т. 4, № 1. - C. 45-51.

7. Shamionov R. M. Adaptational potential and subjective well-being of comprehensive school graduates and first year students of higher educational institutions // Procedia - Social and Behavioral Sciences. - 2014. - Vol. 131. - P. 51-56.

8. ТарасоваЛ. Е. Взаимосвязь характеристик Я-концепции старшеклассников и показателей адаптации к образовательной среде // Современные проблемы науки и образования (электронный журнал). - 2013. - № 4. C. 336. - URL: https://science-education.ru/ru/article/view?id=9860 (дата обращения: 03.12.2016). 
9. Малышев И. В. Взаимосвязь адаптационной готовности личности и эмоционального выгорания у предствителей стрессогенных профессий // Современные исследования социальных проблем (электронный научный журнал). - 2016. - № 8 (64). - С. 123-132. URL: http://journal-s.org/index.php/sisp/article/view/9343/pdf (дата обращения: 10.10.2016).

10. Lopes P. N., Mestre J. M., Guil R., et al. The role of knowledge and skills for managing emotions in adaptation to school: social behavior and misconduct in the classroom // American Educational Research Journal. - 2012. - Vol. 49, Issue 4. - P. 710-742. - DOI: 10.3102/0002831212443077

11. Amiot C. E., Terry D. J., Wirawan D., et al. Changes in social identities over time: The role of coping and adaptation processes // British Journal of Social Psychology. - 2010. - Vol. 49, Issue 4. - P. 803-826. - DOI: 10.1348/014466609X480624

12. Григорьева М. В. Понятийный аппарат психологии адаптации личности // Известия Саратовского университета. Новая серия. Акмеология образования. Психология развития. - 2014. - Т. 3, № 3. С. 259-263.

13. Шамионов Р. М. Соотношение адаптационной готовности и социальной активности личности // Теоретическая и экспериментальная психология. - 2012. - Т. 5, № 2. - С. 72-80.

14. Корнилова Т. В. Принцип неопределенности в психологии выбора и риска // Психологические исследования. - 2015. - T. 8, № 40. - C. 3. - URL: http://psystudy.ru/index.php/num/2015v8n40/1111-kornilova40.html (дата обращения: 08.05.2016).

15. Корнилова Т. В. Ригидность, толерантность к неопределенности и креативность в системе интеллектуально-личностного потенциала человека // Вестник Московского университета. Серия 14: Психология. 2013. - № 4. - С. 36-47.

16. Корнилова Т. В. Толерантность к неопределенности и интеллект как предпосылки креативности // Вопросы психологии. - 2010. - № 5. C. 3-12.

17. Осин Е. Н. Факторная структура русскоязычной версии шкалы общей толерантности к неопределенности Д. МакЛейна // Психологическая диагностика. - 2010. - № 2. - С. 65-86.

18. Осницкий А. К. Определение характеристик социальной адаптации // Психология и школа. - 2004. - № 1. - С. 43-56.

19. Shamionov R. M. Risk Society and Humanity // Chaos, Complexity and Leadership 2014 / S. S. Erçetin (ed.). - New York : Springer, 2016. - P. 209-225. 


\section{References}

1. Nalchadzhyan A. A. Psikhologicheskaya adaptatsiya [Psychological adaptation]. Moscow, Eksmo Publ., 2010. 268 p.

2. Dzhaner'yan S. T., Astaf'eva I. N. Social frustration of school graduates (boys and girls) and their individual psychological features. Aktual'nye problemy gumanitarnykh i estestvennykh nauk, 2015, no. 9-2, pp. 182-186 (in Russian).

3. Shamionov R. M. The association between tolerance to uncertainty and the person's adaptive readiness to change. Vestnik Rossiiskogo universiteta druzhby narodov. Seriya: Psikhologiya i pedagogika - Bulletin of Russian Peoples'Friendship University. Series Psychology and Pedagogics, 2016, no. 3, pp. 28-39 (in Russian).

4. Grigor'eva M. V. The role of social intelligence in senior pupils' psychological adaptation. Teoriya i praktika obshchestvennogo razvitiya - Theory and Practice of Social Development, 2015, no. 6, pp. 171-174 (in Russian).

5. Arendachuk I. V. Personal potential socio-psychological adaptation as a resource of psychological readiness to risk. Izvestiya Saratovskogo universiteta. Novaya seriya. Akmeologiya obrazovaniya. Psikhologiya razvitiya - Izvestiya of Saratov University. New Series. Series: Educational Acmeology. Developmental Psychology, 2015, V. 4, no. 3, pp. 219-226 (in Russian).

6. Bocharova E. E. The person's adaptive readiness to social changes. Izvestiya Saratovskogo universiteta. Novaya seriya. Akmeologiya obrazovaniya. Psikhologiya razvitiya - Izvestiya of Saratov University. New Series. Series: Educational Acmeology. Developmental Psychology, 2015, V. 4, no. 1, pp. 45-51 (in Russian).

7. Shamionov R. M. Adaptational potential and subjective well-being of comprehensive school graduates and first year students of higher educational institutions. Procedia - Social and Behavioral Sciences, 2014, V. 131, pp. 51-56.

8. Tarasova L. E. The relationship between senior pupils' self-concept characteristics and the parameters of adaptation to the educational environment. Modern Problems of Science and Education, 2013, no. 4, p. 336. Available at: https://science-education.ru/ru/article/view?id=9860 (Accessed 3 December 2016).

9. Malyshev I. V. The relationship between the person's adaptive readiness and emotional burnout in representatives of stressful professions. Modern Researches of Social Problems, 2016, no. 8 (64), pp. 123-132. Available at: http://journal-s.org/index.php/sisp/article/view/9343/pdf (Accessed 10 December 2016).

10. Lopes P. N., Mestre J. M., Guil R., et al. The role of knowledge and skills for managing emotions in adaptation to school: social behavior and misconduct in the classroom. American Educational Research Journal, 2012, V. 49, Issue 4, pp. 710-742. DOI: 10.3102/0002831212443077 
11. Amiot C. E., Terry D. J., Wirawan D., et al. Changes in social identities over time: The role of coping and adaptation processes. British Journal of Social Psychology, 2010, V. 49, Issue 4, pp. 803-826. DOI: 10.1348/014466609X480624

12. Grigor'eva M. V. Conceptual apparatus of the psychology of the person's adaptation. Izvestiya Saratovskogo universiteta. Novaya seriya. Akmeologiya obrazovaniya. Psikhologiya razvitiya - Izvestiya of Saratov University. New Series. Series: Educational Acmeology. Developmental Psychology, 2014, V. 3, no. 3, pp. 259-263 (in Russian).

13. Shamionov R. M. The association between adaptive readiness and the person's social activity. Teoreticheskaya i eksperimental'naya psikhologiya - Theoretical and Experimental Psychology, 2012, V. 5, no. 2, pp. 72-80 (in Russian).

14. Kornilova T. V. Uncertainty principle in the psychology of choice and risk. Psychological Research, 2015, V.8, no. 40, p. 3. Available at: http://psystudy.ru/ index.php/num/2015v8n40/1111-kornilova40.html (Accessed 8 May 2016).

15. Kornilova T. V. Rigidity, tolerance to uncertainty, and creativity in the system of the person's intellectual personal potential. Vestnik Moskovskogo universiteta. Seriya 14: Psikhologiya - Moscow University Psychology Bulletin, 2013, no. 4, pp. 36-47 (in Russian).

16. Kornilova T. V. Tolerance to uncertainty and intelligence as preconditions for creativity. Voprosy psikhologii, 2010, no. 5, pp. 3-12 (in Russian).

17. Osin E. N. The factor structure of the Russian version of D. McLane general tolerance to uncertainty scale. Psikhologicheskaya diagnostika - Psychological Diagnostics, 2010, no. 2, pp. 65-86 (in Russian).

18. Osnitskii A. K. Determining the characteristics of social adaptation. Psikhologiya i shkola - Psychology and School, 2004, no. 1, pp. 43-56 (in Russian).

19. Shamionov R. M. Risk society and humanity. Chaos, Complexity and Leadership, 2014, S. S. Erçetin (ed.), New York, Springer, 2016, pp. 209-225. 